\title{
Effect of Diffusion on Lithium Intercalation in Titanium Dioxide
}

\author{
Marina V. Koudriachova, ${ }^{1}$ Nicholas M. Harrison, ${ }^{2}$ and Simon W. de Leeuw ${ }^{1}$ \\ ${ }^{1}$ Department of Applied Physics, Computational Physics, TU Delft, Lorentzweg 1, 2628 CJ Delft, The Netherlands \\ ${ }^{2}$ Department of Chemistry, Imperial College of Science and Technology, London SW7 2AY, United Kingdom \\ and CLRC, Daresbury Laboratory, Daresbury, Warrington WA4 4AD, United Kingdom
}

(Received 3 October 2000)

\begin{abstract}
A new model of $\mathrm{Li}$ intercalation into rutile and anatase structured titania has been developed from first principles calculations. The model includes both thermodynamic and kinetic effects and explains the observed differences in intercalation behavior and their temperature dependence. The important role of strong local deformations of the lattice and elastic screening of interlithium interactions is demonstrated. In addition, a new phase of $\mathrm{LiTiO}_{2}$ is reported.
\end{abstract}

DOI: 10.1103/PhysRevLett.86.1275

PACS numbers: 66.30.-h, 64.70.Kb, 82.45.-h

Transition metal oxides are promising electrode materials in advanced high-energy density batteries [1]. The performance of an electrode depends on its ability to intercalate lithium reversibly into the host lattice. The transition metal oxides have open structures capable of accommodating guest ions and a flexible electronic structure which can accommodate donated electrons and provide sufficient ionic and electronic conductivity. These properties result in a number of low-energy sites for guest ions within the lattice and the potential for high capacity lithium ion intercalation.

Battery performance is usually characterized by low current discharge curves which approximate the equilibrium voltage difference between the electrodes, often referred to as the open circuit voltage (OCV). The key factors in applications are high-energy density and reversible structural changes on $\mathrm{Li}$ intercalation for a large range of the insertion concentration.

For a material in thermodynamic equilibrium the OCV is fully determined by the difference in the chemical potential of lithium between the anode and the cathode which is determined by the ordering of the lithium ions in the host structure. However, in practical applications the OCV is also often limited by lithium ion diffusion which prevents ions from reaching thermodynamically stable sites. The design of new battery materials requires a microscopic understanding of the stable sites and diffusion pathways which is very difficult to obtain from experiment. Therefore, first principles simulation, which provides accurate and reliable energy surfaces, has a key role to play in identifying and characterizing prospective electrode materials.

Previous simulations, based on a combination of first principles energetics and a Monte Carlo treatment of the statistical mechanics, have focused on the thermodynamics of lithium insertion. Thus, phase diagrams and equilibrium OCV's have been computed for $\mathrm{Li}_{x} \mathrm{CoO}_{2}$ [2,3], $\mathrm{Li}_{x} \mathrm{MnO}_{2}$ [4], and $\mathrm{Li}_{1+x} \mathrm{~V}_{3} \mathrm{O}_{8}$ [5]. This methodology requires the representation of configurational energies using effective interaction parameters which are assumed to be valid over a wide range of insertion concentrations.
Another interesting electrode material is nanostructured $\mathrm{TiO}_{2}$ which is currently used in solar cell applications [6]. It has a number of practical advantages as it is readily available, chemically stable, semiconducting, inexpensive, and nontoxic [7]. The most common natural forms of $\mathrm{TiO}_{2}$ are rutile and anatase. Lithium intercalation into both forms has been studied extensively. In $\mathrm{Li}_{x} \mathrm{TiO}_{2}$ the reported maximum electrochemical insertion varies between $x=0.1$ and 1 for rutile [8-11] and 0.5 and 1 for anatase $[10,12-14]$. One likely reason for the large variation in these studies is the effect of temperature. This suggests that diffusion may be an important limiting factor in the performance of rutile and anatase and that unlike cobalt and manganese dioxide, thermodynamic considerations alone are insufficient to determine the OCV. Previous theoretical studies of $\mathrm{Li}_{x} \mathrm{TiO}_{2}$ have concentrated on determining likely intercalation sites and energetics $[15,16]$. However, only a single lithium concentration was considered and the relaxation of the lattice was restricted either by imposing rigid cluster boundary conditions [15] or by considering only isotropic variations of the cell [16]. As is clear from neutron diffraction studies of anatase [17], and will become evident below, unconstrained structural relaxation is vital in these systems.

In the current study fully relaxed first principles simulations have been used to compute the thermodynamics of lithium intercalation in the rutile and anatase structures for a wide range of concentrations and possible configurations. Lithium ion diffusion pathways and barriers have also been determined. The observed shape and temperature dependence of the OCV in both rutile and anatase are discussed in light of these new results.

All calculations were performed within the pseudopotential plane-wave formalism [18,19]. Electron exchange and correlation effects were treated within the spin polarized generalized gradient approximation [20] with ultrasoft pseudopotentials [21] used to replace the Ti $(1 s, 2 s, 2 p)$, O $(1 s)$, and $\mathrm{Li}(1 s)$ core orbitals. The number of Monkhorst-Pack $k$ points was determined from a spacing in the reciprocal space of $0.1 \AA^{-1}$. A plane wave 
cutoff energy of $380 \mathrm{eV}$ was found to converge the total energy to $0.01 \mathrm{eV}$ per formula unit.

Both rutile and anatase are tetragonal crystals with highly anisotropic elastic and dielectric properties. Anatase consists of edge sharing $\mathrm{TiO}_{6}$ octahedra while in rutile octahedra share edges in the $c$ direction but are corner sharing in the $a b$ plane. Between the octahedra there are two potential lithium intercalation sites having either octahedral or tetrahedral oxygen coordination. Initial calculations introducing a single $\mathrm{Li}$ ion into the unit cell $(x=0.5)$ indicated a distinct preference for intercalation into the octahedral site - by $\approx 0.70 \mathrm{eV}$ in rutile and $\approx 0.45 \mathrm{eV}$ in anatase. In what follows calculations have been restricted to intercalation into octahedral sites. Low-energy ordered configurations of $\mathrm{Li}$ ions were sought for a wide range of concentrations $(x=1 / 16,1 / 8,1 / 4,1 / 2,3 / 4,1)$ in both rutile and anatase. For each $x$ a number of periodic supercells were considered and the structure fully relaxed.

First, intercalation into the rutile structure was examined. For $x<1 / 2$ the low-energy ordered configurations favor Li occupation of neighboring octahedra in the $a b$ planes and $\mathrm{Li}$ separation in the $c$ direction. This result is in contrast to expectations based on dielectric screening of $\mathrm{Li}-\mathrm{Li}$ interactions which would favor packing in the $c$ direction. It occurs because the $\mathrm{Li}$ ion strongly distorts the local environment and the distortion is more rapidly screened by the corner sharing octahedra in the $a b$ plane. The elastic screening of the distortion dominates in this instance. This screening mechanism leads to interactions which vary strongly with concentration. This means that the energetics cannot be described using concentration independent interaction parameters and thus it is not convenient to use a Monte Carlo simulation to compute the chemical potential [2]. Instead the excess free energy is approximated from the variation of the total energy with respect to metallic lithium and bulk rutile computed at zero temperature.

The OCV obtained from these data by numerical differentiation is displayed in Fig. 1 and is in agreement with that observed at elevated temperatures. The observed OCV displays steps at $x \approx 1 / 8$ and $x \approx 1 / 2$. The step at $1 / 2$ is $0.1 \mathrm{eV}$ while that at $1 / 8$ has been reported to be $\approx 0.3$ [11] and $\approx 0.1 \mathrm{eV}$ [12]. The majority of the discharge capacity occurs on the plateau between $x=1 / 8$ and $x=1 / 2$. The computed voltage in this region is $1.75 \mathrm{eV}$ which may be compared to the observed values of $1.4-1.5 \mathrm{eV}$ [11,12].

The shape of the OCV curve has been explained previously in terms of a dielectric screening model [12]. The steps in the OCV were related to ordered structures resulting from filled channels in the $c$ direction spaced in the $a b$ plane [12]. However, diffusion in rutile is highly anisotropic and abnormally fast along $c$ [22]. One would therefore expect structures involving $c$ occupancy to be accessible at all temperatures and therefore this model fails to explain the absence of intercalation along $c$ at room temperature $[12,13]$. The interpretation of the OCV resulting from the current work is very different. The initial

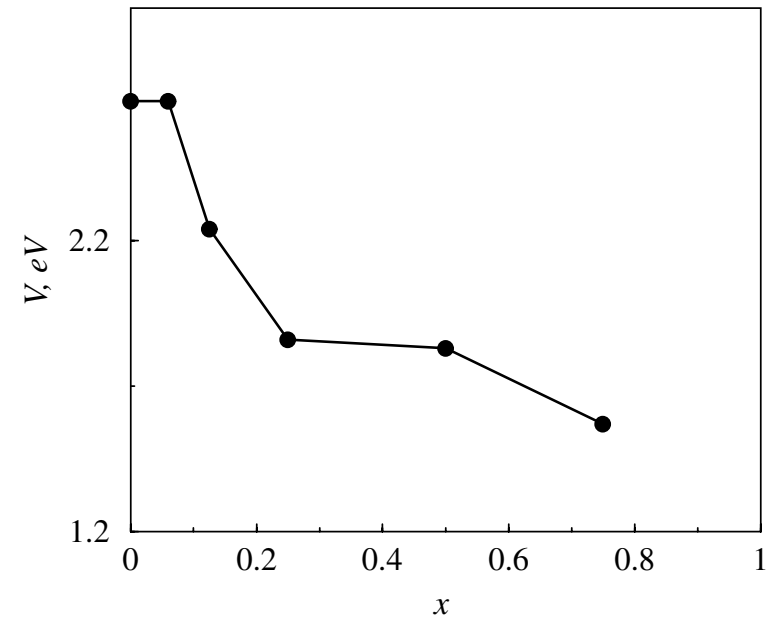

FIG. 1. Calculated OCV profile for $\mathrm{Li}_{x} \mathrm{TiO}_{2}$ rutile.

plateau at $x \leq 1 / 8$ (Fig. 1) corresponds to the coexistence of effectively noninteracting $\mathrm{Li}$ ions in $a b$ planes. At these concentrations 2nd neighbor exclusion in the $a b$ planes is possible and the $\mathrm{Li}$ ions adopt well spaced configurations for which the interactions are fully screened. The screening of these interactions leads to very strong local deformations of the structure and, in particular, in the closing of second neighbor sites. This results in unexpected occupancy patterns at higher concentrations. For instance, at $x>1 / 8$ nearest neighbor sites become occupied before second neighbor sites and it is these configurations which are associated with the observed step in the OCV. The plateau between $x=1 / 8$ and $x=1 / 2$ is due to the gradual increase in the occupancy of the $a b$ planes until at $x=1 / 2$ all sites in the plane are full resulting in a structure containing lithiated $a b$ planes separated in the $c$ direction. As the concentration of $\mathrm{Li}$ ions increases the screening becomes less efficient until at $x=1 / 2$, when it is no longer possible to avoid the occupancy of nearest neighbor sites in the $c$ direction, interactions in the $a b$ and $c$ directions are of a similar magnitude. This results in a small step in the OCV at $x=1 / 2$ as nearest neighbor occupancy in the $c$ direction begins.

The volume expansion up to $x=1 / 2$ is almost exclusively due to the expansion of the Li-containing octahedra. For $x>1 / 2$ the predicted volume increases faster than that expected from local distortions because the packing of the octahedra is disrupted. This is a strong indication that the system will undergo a phase transition. A transition to a currently unidentified structure has been observed at $x=1 / 2[11,12]$ and at $x=3 / 4$ a further transition to a hexagonal structure occurs [11]. This possibility was tested and the latter structure was found to be $\approx 0.26 \mathrm{eV}$ per formula unit more stable than lithiated rutile.

Although it is clear that Li intercalation is thermodynamically favorable the absence of intercalation at room temperature indicates that kinetic restrictions are important. In order to examine this adiabatic energy surfaces for Li diffusion have been computed for $x=1 / 2$ in a single 
rutile unit cell. The energy surface for diffusion along $c$ is displayed in Fig. 2. The fractional coordinate, $z$, is such that at $z=0.5$ the Li ion sits in the center of the octahedral hole. In the symmetry irreducible path $(1 / 4 \leq z \leq 1 / 2)$ minima of roughly equal depth are observed at $z=0.30$ and 0.5 separated by a barrier of $0.04 \mathrm{eV}$. For diffusion in the $a b$ plane the preferred pathway is found to be through the tetrahedral site leading to a barrier which is significantly higher than in the $c$ direction $(\approx 0.8 \mathrm{eV})$. By assuming an attempt frequency of $10^{12} / \mathrm{s}$ diffusion coefficients of $D_{c} \approx 10^{-6} \mathrm{~cm}^{2} / \mathrm{s}$ and $D_{a b} \approx 10^{-15} \mathrm{~cm}^{2} / \mathrm{s}$ are obtained. The observed diffusion is dominated by that in the $c$ direction and is reported to be $D \approx 10^{-6} \mathrm{~cm}^{2} / \mathrm{s}$ [22] in excellent agreement with that computed here. It has also been suggested previously that $D_{a b} \ll D_{c}$ [22]. These data coupled with the strong elastic screening discussed above suggest a simple model of the temperature dependence of intercalation in rutile.

$\mathrm{Li}$ insertion proceeds through rapid diffusion along the $c$ direction. At low temperature the very slow diffusion in the $a b$ planes prevents ions from reaching thermodynamically favorable positions. The relatively poor screening of the $\mathrm{Li}-\mathrm{Li}$ interactions in the $c$ direction leads to rather long range effective repulsive interactions in $c$. At the same time the rapid elastic screening of interactions in the $a b$ plane provides a trapping mechanism for Li-ion pairs (and possibly for larger clusters). Once a pair has formed the $c$ channel is effectively blocked for further intercalation as diffusion in the $a b$ plane is poor. At elevated temperatures in-plane diffusion is considerably enhanced $\left(D_{a b} \approx 10^{-12} \mathrm{~cm}^{2} / \mathrm{s}\right.$ at $\left.120^{\circ} \mathrm{C}\right)$ unblocking the $c$ channels and intercalation proceeds. In fact, at $120^{\circ} \mathrm{C} \mathrm{Li}$ is observed to intercalate up to full loading [11].

A similar analysis indicates that the intercalation of lithium into the anatase structure is also limited by diffusion. Intercalation is observed to proceed as a single phase reaction up to $x=0.05-0.1$ with further insertion producing a two phase equilibrium of Li-poor and Li-rich regions. The Li-rich phase is observed to be an orthorhombic distortion of anatase with composition $\mathrm{Li}_{0.5} \mathrm{TiO}_{2}[17,23]$.

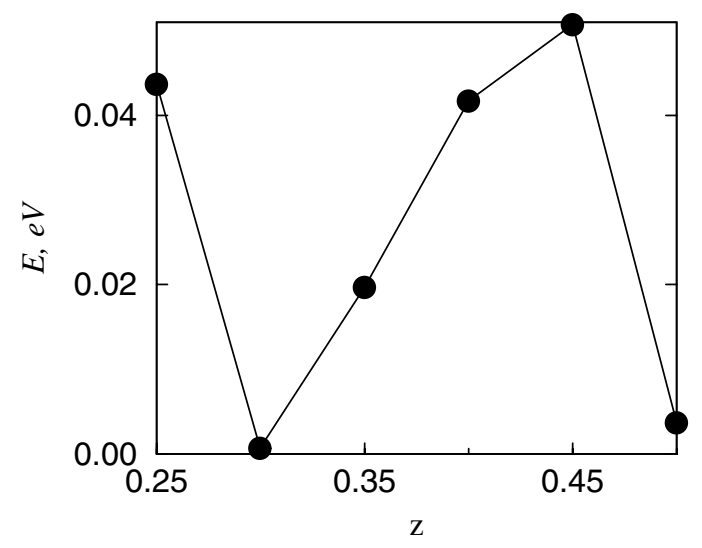

FIG. 2. Relative energy of $\mathrm{Li}_{0.5} \mathrm{TiO}_{2}$ rutile as a function of the Li-ion position.
Low-energy structures for lithiated anatase were sought for $x=1 / 16,1 / 8,1 / 4,1 / 2,3 / 4$, and 1 . At $x=1 / 16$ the Li ions occupy essentially four-coordinated sites at the center of distorted octahedral holes. The lowest energy configurations are with sites well separated and $\mathrm{Li}-\mathrm{Li}$ interactions are effectively screened. At higher concentrations Li-Li interactions become highly anisotropic. The local distortion caused by a Li ion occupying an octahedral site opens the neighboring sites in the $c$ direction while closing those in the $a b$ plane. This "overscreening" in the $c$ direction leads to an effective attraction, while in the $a b$ planes the interactions are highly repulsive. The lowest energy configurations at $x=1 / 8$ and $1 / 4$ correspond to filled channels in the $c$ direction separated in the $a b$ planes.

The computed OCV for anatase is shown in Fig. 3. The shape of the OCV curve is typical of a van der Waals loop and is strongly indicative of a phase separation between a Li-rich and a Li-poor phase as is observed experimentally. Thus, the lowest energy configurations at $x=1 / 8$ and $1 / 4$ are thermodynamically metastable states of the system. This is confirmed by noting that the computed energy of the $x=1 / 4$ state is $\approx 0.15 \mathrm{eV} / \mathrm{Li}$ higher than the coexistence of the stable orthorhombic $(x=1 / 2)$ and the Li-poor $(x=1 / 16)$ phases. The calculations suggest a simple model of intercalation into the anatase structure. Based on the deformations described above diffusion for $x>1 / 16$ can be expected to be highly anisotropic. Diffusion proceeds via nearest neighbor octahedra which have been opened in the $c$ direction but closed in the $a$ and $b$ directions. Thus, fast diffusion along $c$ and slow diffusion in the $a b$ planes are expected. However, once a neighboring octahedron in the $a$ or $b$ direction is occupied the octahedron expands and diffusion proceeds. In practice, $\mathrm{Li}$ insertion is observed to proceed through the (101) surface along the $a$ direction [24]. Slow diffusion in the $b$ direction [perpendicular to the (101) surface] leads to a strong concentration gradient in which $c$ channels are occupied in the near surface region, but diffusion into the bulk is limited. Diffusion then proceeds only by the filling of $c$ channels neighboring in the $a$ or $b$ direction. This leads to a strong tendency for the Li concentration in the near

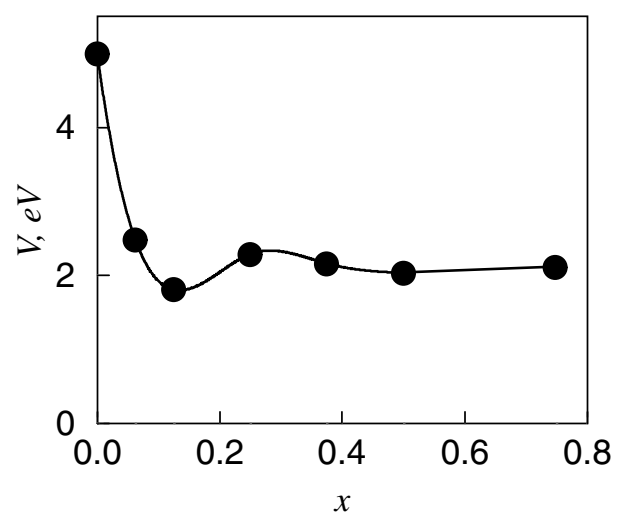

FIG. 3. Calculated OCV profile for $\mathrm{Li}_{x} \mathrm{TiO}_{2}$ anatase. 
surface region to become $x=1 / 2$ and for the orthorhombic phase to form. This provides a natural mechanism for the formation of the thermodynamically stable two phase system. This model implies that there will be an initial barrier to intercalation associated with occupying neighboring $c$ channels as is in fact observed [25]. This two phase $\mathrm{Li}$ insertion must be initiated by the application of an overvoltage [12].

At room temperature the maximum observed insertion ratio is $x=1 / 2$ [12] which, in the current model, corresponds to the completion of the orthorhombic phase. At elevated temperature higher loadings can be achieved. At $x=3 / 4$ and 1 calculations were performed in which three and four octahedral sites per cell were occupied. These configurations are thermodynamically stable, but diffusion is now limited by Li-Li repulsions. The computed diffusion barrier at $x=3 / 4$ is $\approx 1 \mathrm{eV}$, while at $x=1 / 2$ the barrier is $\approx 0.5 \mathrm{eV}$ in excellent agreement with that observed [26]. At $x=1$ the stable structure corresponds to $\mathrm{Li}$ ions occupying the center of the octahedral holes with essentially sixfold coordination in a tetragonally distorted rock-salt lattice. Although this structure has been observed in other transition metal oxides [27] this is, to our knowledge, the first confirmation of its existence in $\mathrm{LiTiO}_{2}$. Within the model presented here slow diffusion out of this structure results in the observed structural aging of anatase when intercalated to high concentrations at high temperature [11].

In conclusion, a new model of $\mathrm{Li}$ intercalation into rutile and anatase structured $\mathrm{TiO}_{2}$ has been developed from first principles calculations. The model includes both thermodynamic and kinetic effects and explains the observed differences in intercalation behavior and their temperature dependence. The absence of insertion into rutile at room temperature is explained in terms of the inaccessability of the low-energy configurations due to highly anisotropic diffusion. In anatase the need for activated insertion and the aging of the system at high concentrations are explained in terms of strong local distortions of the structure. The calculations correctly predict a phase separation in anatase into a $\mathrm{Li}$-rich phase $\left(\mathrm{Li}_{0.5} \mathrm{TiO}_{2}\right)$ and a $\mathrm{Li}$-poor phase. The geometry of orthorhombic $\mathrm{Li}_{0.5} \mathrm{TiO}_{2}$ is computed for the first time and we predict the existence of a new distorted rock-salt phase for $\mathrm{LiTiO}_{2}$. The model developed here has a number of consequences for the design of new battery materials. For instance, aging in anatase can be avoided by restricting the insertion fraction and efficient insertion is much more likely to be achieved along the $c$ direction than the $a$ direction as in current devices.

This work was supported by The Netherlands Foundation for Fundamental Research of Matter (FOM) as part of the programme for Massively Parallel Computing (MPR). The use of the Cray-T3E at the CSAR service in Manches- ter was funded through the EPSRC's High Performance Computing Initiative within the U.K. Car-Parrinello consortium (GR/N02337).

[1] D. O'Hare, in Inorganic Materials, edited by D. W. Bruce and D. O'Hare (John Wiley \& Sons, Ltd., Chichester, UK, New York, 1996), pp. 171-254.

[2] C. Wolverton and A. Zunger, Phys. Rev. Lett. 81, 606 (1998).

[3] A. Van der Ven, M. K. Aydinol, G. Ceder, G. Kresse, and J. Hafner, Phys. Rev B 58, 2975 (1998).

[4] S. K. Mishra and G. Ceder, Phys. Rev. B 59, 6120 (1999).

[5] R. Benedek, M. M. Thackeray, and L. H. Yang, Phys. Rev. B 60, 6335 (1999).

[6] B. O'Regan and M. Grätzel, Nature (London) 353, 737 (1991).

[7] S. Huang, L. Kavan, A. Kay, and M. Grätzel, J. Electrochem. Soc. 141, 142 (1995).

[8] M. Voinov, Proc. Electrochem. Soc. 81, 352-357 (1981).

[9] F. Bonino, L. Busani, M. Manstretta, B. Rivolta, and A. B. Scrosatti, J. Power Sources 14, 261 (1985).

[10] T. Ohzuku, Z. Takehara, and S. Yoshizawa, Electrochim. Acta 27, 1263 (1982).

[11] W. J. Macklin and R. J. Neat, Solid State Ion. 53-56, 694-700 (1992).

[12] B. Zachau-Christiansen, K. West, and S. Atlung, Solid State Ion. 28-30, 1176-1182 (1988).

[13] F. Bonino, L. Busani, M. Lazzari, M. Mantretta, and B. Scrosatti, J. Power Sources 6, 261 (1981).

[14] T. Ohzuku and T. Kodama, J. Power Sources 14, 153 (1985).

[15] A. Stashans, S. Lunell, R. Bergström, A. Hagfeldt, and S-E. Lindquist, Phys. Rev. B 53, 159 (1996).

[16] W. C. Mackrodt, J. Solid State Chem. 142, 428-439 (1999).

[17] R. J. Cava, D. W. Murphy, S. Zahurak, A. Santorio, and R. S. Roth, J. Solid State Chem. 53, 64 (1984).

[18] M. C. Payne, M. P. Teter, D. C. Allan, T. A. Arias, and J. D. Joannopoulos, Rev. Mod. Phys. 64, 1045 (1992).

[19] CASTEP 3.9 Academic version, licensed under the UKCP-MSI agreement, 1999.

[20] J. P. Perdew, Phys. Rev. B 34, 7406(E) (1986).

[21] D. Vanderbilt, Phys. Rev. B 41, 7892 (1990).

[22] O. W. Johnson, Phys. Rev. A 136, 284 (1964).

[23] D. W. Murphy, R. J. Cava, S. M. Zahurak, and A. Santoro, Solid State Ion. 9-10, 4, 13 (1983).

[24] H. O. Finklea, in Semiconductor Electrodes, Studies in Physical and Theoretical Chemistry (Elsevier, Amsterdam, 1988), Vol. 55, pp. 43-145.

[25] L. Kavan, M. Grätzel, S. E. Gilbert, C. Klemenz, and H. J. Scheel, J. Am. Chem. Soc. 118, 6716-6723 (1996).

[26] R. Van der Krol, A. Goossens, and E. Meulenkamp, J. Electrochem. Soc. 146, 3150 (1999).

[27] T. A. Hewston and B. L. Chamberland, J. Phys. Chem. Solids 48, 97 (1987). 